\title{
The Role of NT-proBNP as Prognostic Biomarker for Preeclampsia Complications: A Systematic Review Protocol
}

Aiman Hilmi Asaduddin ( $\sim$ aimanhilmi02@student.uns.ac.id)

Faculty of Medicine, Universitas Sebelas Maret https://orcid.org/0000-0002-2518-1800

Shafira Yasmine Anshari

Faculty of Medicine, Universitas Sebelas Maret

Agni Shalha Ali

Faculty of Medicine, Universitas Sebelas Maret

Ardhia Fefrine Indarta

Faculty of Medicine, Universitas Sebelas Maret

Muhammad Adrianes Bachnas

Department of Obstetric and Gynecology, Faculty of Medicine, Universitas Sebelas Maret

Ratih Puspita Febrinasari

Department of Pharmacology, Faculty of Medicine, Universitas Sebelas Maret

Vitri Widyaningsih

Department of Public Health, Faculty of Medicine, Universitas Sebelas Maret

\section{Method Article}

Keywords: NT-proBNP, Preeclampsia, Complications

Posted Date: April 19th, 2021

DOl: https://doi.org/10.21203/rs.3.rs-388263/v1

License: (9) (i) This work is licensed under a Creative Commons Attribution 4.0 International License.

Read Full License 


\section{Abstract}

\section{Background}

There are two central dogmas between the pathophysiology of preeclampsia, the placental origin and the extra-placental origin. Recent findings found that women with a history of preeclampsia are at higher risk of cardiovascular disease and death because of cardiovascular disease. This finding leads to the extraplacental origin. During normal pregnancy, the maternal cardiovascular system undergoes modification. However, hemodynamic and vascular modification are disrupted in women who develop preeclampsia. NT-proBNP is a biomarker that indicates the disruption of the cardiovascular system and can predict the complication of preeclampsia. Based on that information, we would like to investigate the roles of NTproBNP plasma levels in predicting maternal and fetal complications of preeclampsia women. Besides, we also investigate the roles of NT-proBNP plasma levels in predicting the severity and onset of preeclampsia women and the sensitivity and specificity of NT-proBNP plasma levels in preeclamptic women.

\section{Methods}

We designed and registered a study protocol for a systematic review. This study aims to investigate the roles of NT-proBNP plasma levels in predicting maternal and fetal complications of preeclampsia women. The Cochrane Handbook for Systematic Reviews of Diagnostic Test Accuracy was used to structure our methodological approach and the Preferred Reporting Items for a Systematic Review and Meta-Analysis Protocols guidelines in reporting the findings of this review. We will search literature in Hinari, Cochrane Library, ScienceDirect, and Scopus database. Then, we will search the grey literature in OCLC'S OAISTER. Randomized controlled trials and observational studies will be included in the analysis. Three reviewers will independently screen articles, extract data, and evaluate for quality and bias using Newcastle-Ottawa Scale. Studies were included according to the following procedures: (1) Identification, Duplication of articles and non-original research articles will be excluded; (2) Title and Abstract Screening, RCT and observational studies and focused on NT-proBNP levels in preeclampsia; (3) Full-text availability; and (4) Full-text screening based on PICO criteria. Each outcome is synthesized by a qualitative or narrative approach. The results will be divided into certain points, namely (a) Selection of the studies, (b) Characteristics of the Eligible Studies, (c) Data Extraction of the Eligible Studies, and (d) Risk of Bias Analysis Results.

\section{Discussion}

A systematic review of the role of NT-proBNP as predicting biomarkers for preeclampsia complications will be conducted. This study can help predict the severity, onset, and complication of a pregnant woman with preeclampsia.

\section{Background}


Preeclampsia is a complication of pregnancy manifesting as hypertension and proteinuria. It is responsible for around $14 \%$ of global maternal deaths and is a leading cause of morbidity and mortality in pregnant women (1). Clinically, the American College of Obstetrics and Gynecology (ACOG) defines preeclampsia as the presence of hypertension and proteinuria or severe features that occur at more than 20 weeks of gestation in a previously normotensive patient (2). Based on the severity and type of symptoms, preeclampsia can be divided into mild and severe forms. The mild form of preeclampsia is characterized by systolic blood pressure $\geq 140 \mathrm{mmHg}$ or diastolic blood pressure $\geq 90 \mathrm{mmHg}$, and proteinuria $>300 \mathrm{mg} / 24$ hours (3). Meanwhile, severe forms of preeclampsia are characterized by severe hypertension (systolic blood pressure> $160 \mathrm{mmHg}$ or diastolic blood pressure> $110 \mathrm{mmHg}$ ), or severe proteinuria (> $2 \mathrm{~g} / 24$ hours), or signs and symptoms of target organ damage (4). Based on the disease onset, preeclampsia has two subtypes, early-onset and late-onset preeclampsia. Early-onset preeclampsia occurring before or at 33 weeks gestation, and late-onset preeclampsia occurs at 34 weeks gestation or later (5).

The etiology of preeclampsia remains elusive, but there are two colliding central dogma. The two central dogma of preeclampsia etiology is the placental origin and the extra-placental origin. The placental origin is caused by abnormal placentation and is followed by an excess of antiangiogenic factors (2). Abnormal placentation is characterized by the triad of inadequate placentation, placental insufficiency, and vascular reactivity cascade, which caused placental ischemia (6). Antiangiogenic factors and inflammatory cytokines are produced due to placental ischemia, and then they cause systemic vascular dysfunction. Systemic vascular dysfunction can cause proteinuria, hypertension, coagulation abnormalities, and preeclampsia complications such as eclampsia and HELLP syndrome (2). The drawback of this theory is that it fails to explain the postpartum cardiovascular complication of preeclampsia women.

Research has shown an association between preeclampsia and cardiovascular complication during pregnancy or later life. This finding leads to the extra-placental origin of preeclampsia. During pregnancy, the maternal cardiovascular system undergoes modification. However, hemodynamic and vascular modification are disrupted in women who develop preeclampsia. Placental dysfunction occurs as a result of maternal cardiovascular maladaptation in pregnancy (7). The concept of preeclampsia as a placental dysfunction caused by maternal disorders is considered clinically similar to gestational diabetes. Both of them are caused by pregnancy and cured by birth (8). The extra-placental origin theory is possible because the placenta function depends on the need for adequate perfusion from the maternal circulation.

NT-proBNP is a BNP inactive form produced by cardiomyocytes due to heart muscle stretching and an increase of afterload volume (9). The NT-proBNP level increases in line with the increase of left ventricular diastolic dysfunction (9). Increased levels of NT-proBNP reflect ventricular stress and subclinical cardiac dysfunction (9). The NT-proBNP level is significantly increased in preeclampsia women compared to patients with normal pregnancy (10). The NT-proBNP level also continues to increase during the 3 to 6 months postpartum (11). This makes NT-proBNP can be used as a biomarker to screen for preeclampsia cardiovascular complication before and after labor. 


\section{Methods/design}

This is a protocol for a systematic review on the prognostic of NT-proBNP plasma levels in predicting the severity, onset, and complication of preeclampsia women. We will adhere to Preferred Reporting Items for Systematic Reviews and Meta-analysis Protocol (PRISMA-P) in reporting the findings of this review.

\section{Objectives}

\section{Objectives}

\section{Primary objective}

To investigate the roles of NT-proBNP plasma levels in predicting maternal and fetal complications of preeclampsia women.

\section{Secondary objective}

To investigate the roles of NT-proBNP plasma levels in predicting the severity and onset of preeclampsia women, and the sensitivity and specificity of NT-proBNP plasma levels in preeclamptic women.

\section{Criteria for studies included in this review}

\section{Type of studies}

We will include all reports on NT-proBNP plasma levels in predicting preeclampsia complications among maternal and fetal. Moreover, the study will be conducted by observational (cohort, cross-sectional, or case-control) studies or secondary analysis of randomized controlled trials.

\section{Participants}

The target participants are as follows:

- Preeclampsia patients with HELLP syndrome, pulmonary edema, and poor cardiac function. - Fetal complications of preeclampsia (IUGR and prematurity)

\section{Index test}

The index tests are plasma NT-proBNP assays using any method. NT-proBNP is an index widely used to evaluate cardiac function (11). Elevated NT-proBNP plasma levels are associated with preeclampsia and its complication (11).

\section{Search methods used to identify studies}

\section{Electronic searches}


We will search on Hinari, Cochrane Library, ScienceDirect, Scopus, and OCLC's OAISTER (for the grey literature) to identify all observational studies or randomized controlled trials. We restrict the language publication, only English, and with a time restriction of at least 15 years (April 2006 - April 2021). We have outlined the search strategy in Appendix 1.

\section{Data collection and analysis}

\section{Selection of studies}

The selection process will be done by three independent reviewers, and studies were included according to the following procedures: (1) Identification, Duplication of articles and non-original research articles will be excluded; (2) Title and Abstract Screening, RCT and observational studies and focused on NTproBNP levels in preeclampsia; (3) Full-text availability; and (4) Full-text screening based on PICO criteria. The PICO criteria are:

- Patient: Pregnant women diagnosed with preeclampsia

- Indicator: NT-proBNP plasma level

- Comparison: Low vs. High NT-proBNP plasma level

- Outcome: Severity, Onset (early or late), and Complications (Maternal \& Fetal) of Preeclampsia

\section{Data extraction and management}

The general information including the first author's name, published year, country, the number of patients, patient age, pregnancy age, sample extraction time, time of delivery, preeclampsia severity or onset, complications occurred, and level, specificity, sensitivity, and/or cut-off-point of NT-proBNP plasma, and fetal birth weight. Data were extracted using Microsoft Excel (Tabulation method) from study results graphs or tables. Three independent reviewers did data extraction. The quality of the included study was assessed with the Newcastle-Ottawa Scale (NOS) tool (12).

\section{Assessment of methodological quality}

Three investigators will independently evaluate and report the risk of bias using the NOS tool (12). We will assess three domains for the risk of bias: selection, comparability, and outcome. The three domains will contain a total of 9 scoring checklists (12).

\section{Statistical analysis and data synthesis}

Each outcome is synthesized by a qualitative or narrative approach. The results will be divided into certain points, namely (a) Selection of the studies, (b) Characteristics of the Eligible Studies, (c) Data Extraction of the Eligible Studies, and (d) Risk of Bias Analysis Results.

\section{Discussion}


This systematic review will summarize existing knowledge on the role of NT-proBNP as a biomarker for predicting the severity, onset, and complication of preeclampsia among pregnant women. Any modification made to our protocol during the review will be reported in the final paper. We plan to submit the review in a peer-reviewed journal. This protocol can help physicians select an alternative way for predicting maternal and fetal complications of preeclampsia women.

\section{Abbreviations}

ACOG: American College of Obstetrics and Gynecology

BNP: Brain Natriuretic Peptide

HELLP: Haemolysis Elevated Liver Enzymes Low Platelet

IUGR: Intra Uterine Growth Restriction

NOS: Newcastle-Ottawa Scale

NT-proBNP: N-Terminal pro-Brain Natriuretic Peptide

PICO: Patient, Index, Comparison, Outcome

PRISMA-P: Preferred Reporting Items for Systematic Reviews and Meta-Analysis Protocol

\section{Declarations}

\section{Ethics approval and consent to participate}

Not applicable.

\section{Consent for publication}

Not applicable.

\section{Availability of data and materials}

Not Available

\section{Competing interests}

The authors declare that they have no competing interests.

\section{Funding}

The authors did not receive any funding for this study. 
Authors' contribution

\section{Authors' contributions}

AHA and SYA were major contributors in writing the manuscript. All authors contributed to developing the search strategy, the selection criteria, the bias assessment strategy, and data extraction criteria. All authors also read, provided feedback, and approved the final manuscript.

\section{Acknowledgments}

Not Applicable.

\section{References}

1. Leon LJ, Mccarthy FP, Direk K, Gonzalez-Izquierdo A, Prieto-Merino D, Casas JP, et al. Preeclampsia and Cardiovascular Disease in A Large UK Pregnancy Cohort of Linked Electronic Health Records. Circulation. 2019;September(140):1050-60.

2. Rana S, Lemoine E, Granger JP, Karumanchi SA. Preeclampsia: Pathophysiology, Challenges, and Perspectives. Circ Res. 2019;March(124):1094-112.

3. Dhariwal NK, Lynde GC. Update in the Management of Patients with Preeclampsia. Anesthesiol Clin [Internet]. 2017;35(1):95-106. Tersedia pada: http://dx.doi.org/10.1016/j.anclin.2016.09.009

4. Peres GM, Mariana M, Cairrao E. Pre-Eclampsia and Eclampsia: An Update on the Pharmacological Treatment Applied in Portugal †. J Cardiovasc Dev Dis. 2018;5(5):1-13.

5. Lisonkova S, Joseph KS. Incidence of Preeclampsia: Risk Factors and Outcomes Associated with Early- Versus Late-onset Disease. Am J Obstet Gynecol [Internet]. 2013;209(6):544.e1-544.e12. Tersedia pada: http://dx.doi.org/10.1016/j.ajog.2013.08.019

6. Thilaganathan B, Kalafat E. Cardiovascular System in Preeclampsia and Beyond. 2019;522-31.

7. Thilaganathan B. Pre-eclampsia and The Cardiovascular-placental Axis. Ultrasound Obstet Gynecol. 2018;51(6):714-7.

8. Perry H, Khalil A, Thilaganathan B. Preeclampsia and The Cardiovascular System: An Update. Trends Cardiovasc Med [Internet]. 2018;28(8):505-13. Tersedia pada:

https://doi.org/10.1016/j.tcm.2018.04.009

9. Giannubilo SR, Pasculli A, Tidu E, Biagini A, Boscarato V, Ciavattini A. Relationship between Maternal Hemodynamics and Plasma Natriuretic Peptide Concentrations during Pregnancy Complicated by Preeclampsia and Fetal Growth Restriction. J Perinatol [Internet]. 2017;37(5):484-7. Tersedia pada: http://dx.doi.org/10.1038/jp.2016.264

10. Kim HM, Choo YS, Seong WJ. Serum NT-proBNP Levels as A Marker for Cardiopulmonary Function in Preeclampsia. Clin Exp Obstet Gynecol. 2020; XLVII(4):511-5.

11. Kumari S, Gupta M, Vardhan H, Saini V, Gupta S. NT-proBNP: A Biochemical Marker of Maternal Complications in Preeclampsia. J Clin Diagnostic Res. 2017;11(11):12-6. 
12. Wells G, Shea B, O'Connell D, Peterson J, Welch V, Losos M, et al. The Newcastle-Ottawa Scale (NOS) for Assessing The Quality of Nonrandomised Studies in Meta-Analyses [Internet]. Ottawa Hospital Research Institute. 2014 [dikutip 11 April 2021]. Tersedia pada:

http://www.ohri.ca/programs/clinical_epidemiology/oxford.asp\#: :text=The Newcastle-Ottawa Scale

\section{Supplementary Files}

This is a list of supplementary files associated with this preprint. Click to download.

- Appendix1.SearchStrategy.pdf

- Appendix2.ProtocolPRISMA2020Checklist.pdf

- Appendix3.ProtocolPRISMA2020AbstractChecklist.pdf 